\title{
Separate Development of Nitric Oxide Synthase- and Vasoactive Intestinal Polypeptide-Immunoreactive Nerves Arising from the Vertebral Artery in the Rat
}

\author{
Koichi ANDO ${ }^{1)}$, Souta YOSHIDA ${ }^{1)}$, Hitoshi YAMAUCHI ${ }^{1)}$, Shinji $\mathrm{KUBO}^{1)}$ and Haruo KUSABA ${ }^{2)}$ \\ ${ }^{1)}$ Biological Laboratory, Department of Applied Chemistry and Biochemistry, Faculty of Engineering, Kyushu Sangyo University, Higashi- \\ ku, Fukuoka 813-8530 and 2) Muromi Animal Hospital, 1-11-9 Muromi, Sawara-ku, Fukuoka 814-0015, Japan
}

(Received 8 July 2005/Accepted 24 October 2005)

\begin{abstract}
Development of nitric oxide synthase (NOS)- and vasoactive intestinal polypeptide (VIP)-immunoreactive (-IR) nerves supplying the basilar and vertebral arteries (BA and VA) was investigated in White Wistar rats, using double immunohistochemistry. NOSIR and VIP-IR nerves via the anterior circulation (AC), which mostly expressed $\mathrm{NO}^{+} / \mathrm{VIP}^{+}$, extended to the $\mathrm{BA}$ during the second postnatal week, and usually reached as far as the rostral two third of the BA on PND 20. NOS-IR nerves were completely lack in the cBA and the VA on PND10, and often absent from these arterial regions even at PND 20. Nevertheless, a small number of $\mathrm{VIP}^{+} / \mathrm{NOS}^{-}$nerves were localized in the walls from the caudal BA (cBA) to the VA on PND 5. On PND 20, they frequently met with the descending NOSIR and VIP-IR nerves via the AC around the lower portion of the middle BA. Fiber bundles containing NOS ${ }^{+} / \mathrm{VIP}^{+}$axons were first visualized on the caudal VA at PND 30 and observed frequently at PND 80, with a distinct increase in number of NOS-IR and VIP-IR nerves supplying the cBA and the VA. Thus, NOS-IR nerves coming from the VA develop through its own characteristic sequence that lags markedly behind the time of appearance for VIP-IR nerves from the same vascular route and for NOS-IR and VIP-IR nerves via the AC.
\end{abstract}

KEY WORDS: cerebrovascular innervation, development, NOS-IR nerves, rat, VIP-IR nerves.

J. Vet. Med. Sci. 68(2): 125-130, 2006

Nitric oxide (NO), which is synthesized from L-arginine by the constitutive NO synthase (NOS) in both central and peripheral neurons, has been characterized as a vital neurotransmitter for vasodilator action of cerebral circulation $[6,8,10,15]$. Indeed, the rich innervation of nitrergic nerves, which can be visualized using immunohistochemistry for NOS or histochemistry for nicotinamide adenine dinucleotide phosphate diaphorase (NADPHd), has been demonstrated for the cerebral arterial system in a variety of mammals $[1,4,7,9,12,11]$. Some of these studies, in conjunction with ganglionectomy and/or retrograde tracing techniques, has also confirmed that cerebral perivascular nitrergic nerves, as well as the nerves immunoreactive for vasoactive intestinal polypeptide (VIP), a vasodilatory neuropeptide related to the cerebral circulation, have their major source at the sphenopalatine ganglion (SPG). Further, it has been made clear that NOS immunoreactivity or NADPHd activity coexists with VIP immunoreactivity in the same cerebral perivascular parasympathetic axons $[1,7$, $11,17]$.

Our recent study has accumulated additional information on the rat cerebrovascular nitrergic innervation, that is, NOS-IR nerves appear along the internal ethmoidal artery (IEA) before birth as fiber bundles [3]. The immunoreactive nerves from here usually extend as far as the rostral two third of the basilar artery (BA) at the beginning of the third postnatal week. This finding is in good agreement with the developmental pattern of VIP-IR nerves from the same vascular route [2]. It has also provided another finding that the caudal part of the BA (cBA) and the vertebral artery (VA) are generally devoid of nerves with detectable level of NOS immunoreactivity before postnatal day (PND) 15. This finding does not coincide with the developmental pattern of VIP-IR neuronal projection from the VA to the BA, of which the appearance has been ascertained at PND 3. In order to hold more precisely the pattern of NOS-IR and VIPIR innervations in the rat cerebral arterial tree, we investigated the colocalization of these two neurochemical substances in the cerebral perivascular nerves supplying the rat BA and VA from PND 5 to 80, using double immunostaining.

\section{MATERIALS AND METHODS}

Postnatal White Wistar rats of both sexes, ranging from 5 to 80 days after birth, were used in this study. The day of birth was regarded as postnatal day one (PND1). Mother and newborn rats were bred in the same cage until PND 20, under a room environment kept at constant temperature (23 $\left.\pm 1^{\circ} \mathrm{C}\right)$ and humidity $(48 \pm 3 \%)$, and then housed separately. Food and water were provided ad libitum. The animals were divided by age into the following six groups: PND5, PND10, PND15, PND20, PND30 and PND 80. For each of these groups, seven animals or more were examined for double immunohistochemistry for NOS and VIP. All experiment procedures were conducted in accordance with the National Research Council (NRC) publication guide for care and use of Laboratory Animals (copyright 1996, National Academy of Science).

Tissue preparation: Animals were anesthetized with ethyl ether and perfused via the ascending aorta with cold Ringer's solution, followed by an appropriate volume of Zamboni's fixative. The brains were removed immediately from the skulls, and then postfixed in the same fixative for 

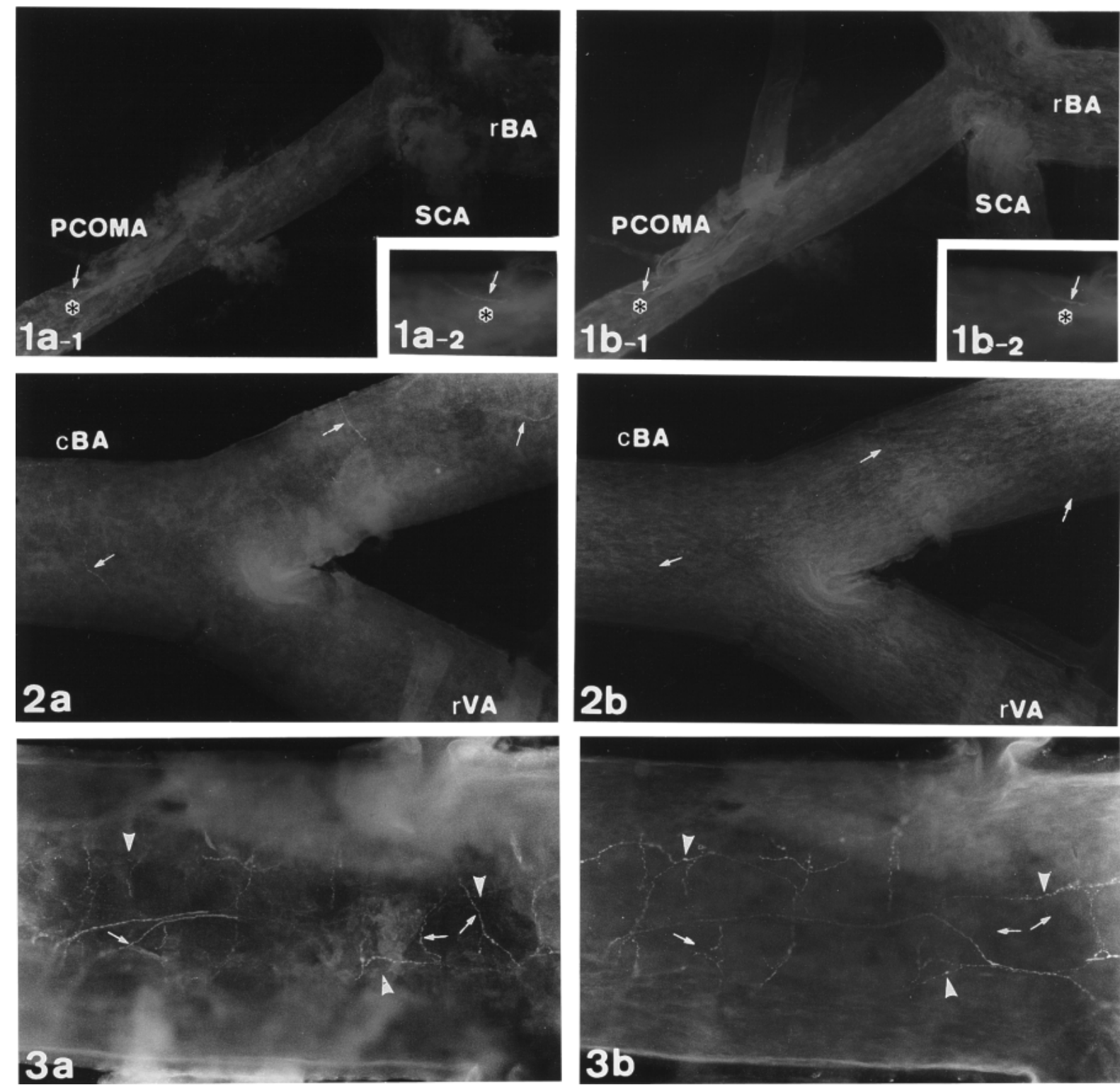

Figs. 1-3. Fluorescence photomicrographs of whole mounts with double staining for VIP (a) and NOS (b) in the major arteries of the posterior circulation on PND 5 (Figs. 1 and 2) and 10 (Fig. 3). Figure 1. Posterior communicating artery (PCOMA), superior cerebellar artery (SCA) and rostral part of the basilar artery (rBA). Arrows indicate nerves immunoreactive for both VIP and NOS. Asterisks indicate the same portion of the PCOMA. $\times 65(\mathrm{a}-1, \mathrm{~b}-1), \times 125(\mathrm{a}-2, \mathrm{~b}-2)$. Figure 2. Caudal part of the BA $(\mathrm{cBA})$ and rostral part of the VA (rVA). Arrows indicate VIP-IR nerves negative for NOS. $\times 65$. Figure 3. Rostral part of the BA. Arrows indicate VIP-IR nerves negative for NOS. Arrowheads indicate NOS-IR nerves negative for VIP. $\times 100$.

$18-24 \mathrm{hr}$ at $4{ }^{\circ} \mathrm{C}$. The cerebral arterial trees were rapidly dissected out from the brains, washed thoroughly with cold $0.01 \mathrm{M}$ phosphate-buffer (PB, pH 7.2). The materials were placed in $0.01 \mathrm{M}$ PB containing $10 \%$ sucrose for one day at $4^{\circ} \mathrm{C}$, and then stored at $4{ }^{\circ} \mathrm{C}$ in phosphate-buffered saline (PBS, pH 7.2) containing 0.3\% Triton X-100 (PBST) over one night.

Immunohistochemical protocol: Whole-mounts of the cerebral arterial trees from each age group were processed for double staining by indirect immunofluorescence as described previously [1]. The whole-mount preparations were incubated for 3 days at $4^{\circ} \mathrm{C}$ in rabbit polyclonal NOS antiserum (Code no. 61-7000; Zymed Lab., San Francisco, U.S.A.) at a dilution of 1:100, and subsequently in guineapig polyclonal VIP antiserum (Code no. B-GP 341-1; EuroDiagnostica, Malmo, Sweden) at a dilution of 1:1000. After washing with PBST, they were incubated for $2 \mathrm{hr}$ at $20^{\circ} \mathrm{C}$ in donkey anti-rabbit IgG conjugated with fluorescein isothio- 

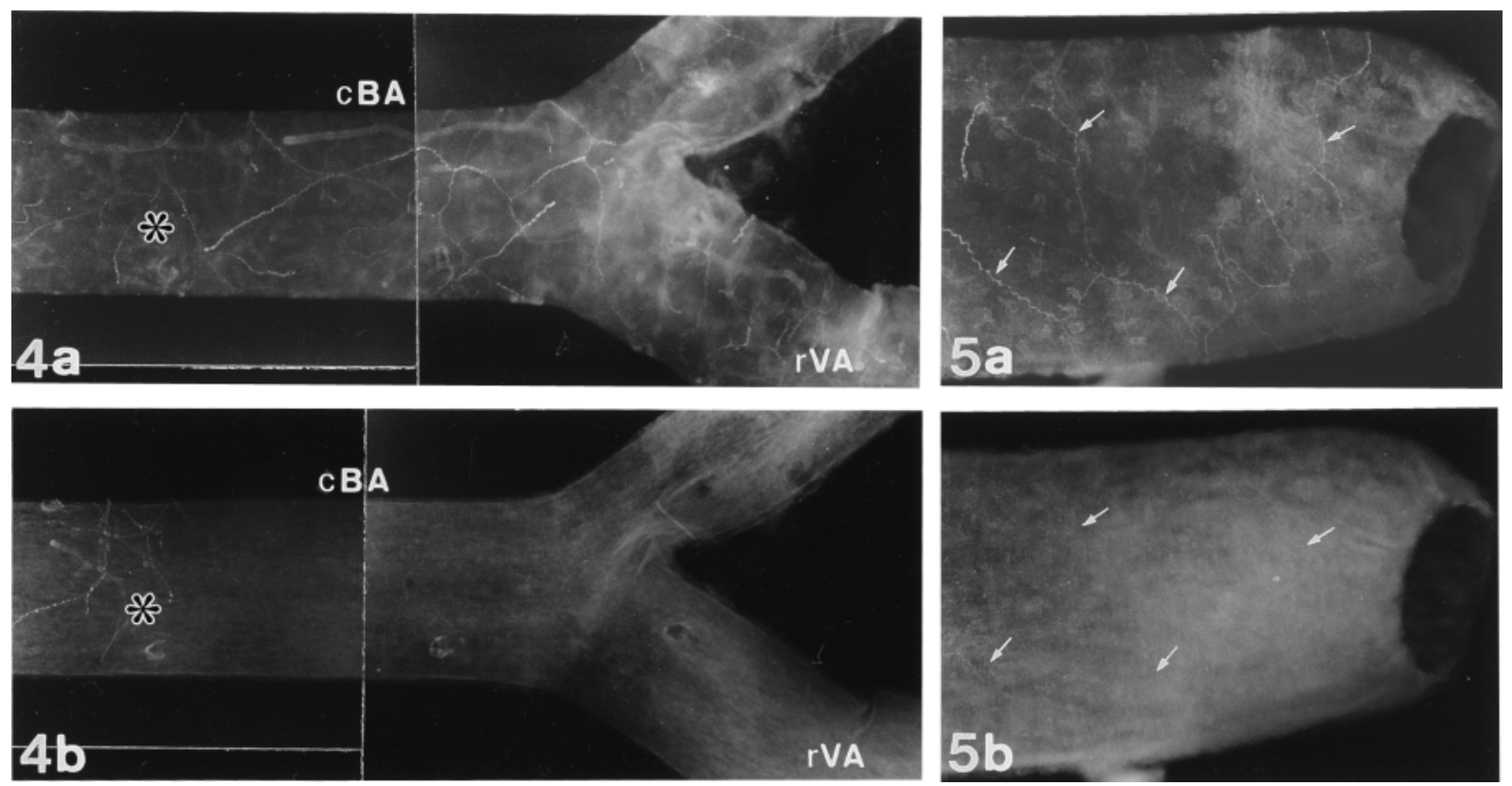

Figs. 4, 5. Fluorescence photomicrographs of whole mounts with double staining for VIP (a) and NOS (b) in the basilar and vertebral arteries (BA, VA) on PND 20. Figure 4. Caudal part of the BA (cBA) and rostral part of the VA (rVA). Note the presence of VIP-IR and NOS-IR nerves in the upper portion of the cBA (asterisks) and the absence of NOS-IR nerves in its lower portion and the VA. Note that VIP-IR nerves in the walls from the lower cBA to the VA are all negative for NOS. $\times 50$. Figure 5 . Caudal part of the VA. Arrows indicate VIP-IR nerves negative for NOS. Note the complete lack of NOS-IR nerves with or without VIP immunoreactivity. $\times 100$.

cyanate (FITC, Jackson, Baltimore, U.S.A.) at the dilution of 1:100, and subsequently in donkey anti-guinea pig IgG conjugated with Texas Red (TR, Jakson, Baltimore, U.S.A.) at the dilution of 1:100. The specimens were washed with PBS and mounted in glycerin-PB mixture (1:1), and examined under an Olympus fluorescence microscope equipped with B-dichromic mirror unit (U-MNIB2) and excitation filter (BP 470-490) for FITC and TR-dichromic mirror unit (U-MWIY2) and excitation filter (BP 545-580) for TR. Colocalization of NOS and VIP immunoreactivities in the same cerebral perivascular nerves was archived by alternation of the dichromic mirror filter combination for FITC and TR.

\section{RESULTS}

As reported previously [2, 3], NOS-IR and VIP-IR nerves, which arose from the fiber bundles on the wall of the IEA, spread over all the major arteries of the anterior circulation on PND 5. Caudally oriented nerves from this vascular route, together with those via the cerebral carotid artery (CCA), also projected to the posterior circulation through the posterior ramus (PR), and sometimes penetrated into the posterior communicating artery (PCOMA) (Fig. 1). At this early stage of development, there were no nerves immunoreactive for NOS along the entire length of the BA and VA. Nevertheless, it could very often be observed that a small number of nerves, which were stained positively for VIP but negatively for NOS $\left(\mathrm{VIP}^{+} / \mathrm{NOS}^{-}\right)$, were distributed dispersedly along the walls from the cBA to the VA (Fig. 2).

By PND 10, NOS-IR and VIP-IR nerves descending the $\mathrm{PR}$ from the anterior circulation advanced into the rostral part of the BA through the PCOMA, and grew progressively toward the caudal direction during the second postnatal week. Most of these immunoreactive nerves were stained positively for both NOS and VIP $\left(\mathrm{NOS}^{+} / \mathrm{VIP}^{+}\right)$, and a few expressed either NOS $\left(\mathrm{NOS}^{+} / \mathrm{VIP}^{-}\right)$or $\mathrm{VIP}\left(\mathrm{VIP}^{+} / \mathrm{NOS}^{-}\right)$ (Fig. 3). At this stage, the caudal part of the BA (cBA) and the VA still had no NOS-IR nerves. NOS-IR and VIP-IR nerves via the PCOMA usually attained to the middle part of the BA (mBA) on PND 15, and extended further as far as the level of its rostral two third on PND 20 (Fig. 4), with a significant rise of nerve density. It was occasionally observed at PND 15 and frequently at PND20 that these descending nerves met with the ascending $\mathrm{VIP}^{+} / \mathrm{NOS}^{-}$nerves via the $\mathrm{VA}$ around the lower portion of the mBA. At this point of development, the whole length of the BA and VA was systematically supplied with VIP-IR nerves. Unlike VIP-IR innervation, the cBA from most of the individuals at PND 15 received no supply of $\mathrm{NOS}^{+} / \mathrm{VIP}^{+}$or $\mathrm{NOS}^{+} / \mathrm{VIP}^{-}$nerves. Likewise, the VA was still devoid of NOS-IR nerves, except for only a few axons that were very rarely seen in its rostral part. Even at the PND 20, nerves immunoreactive for NOS were often lack in the cBA and the rostral part of the VA (rVA) or sporadic if they exist (Fig. 4), and the caudal part of the VA (cVA) was equipped with no NOS-IR nerves in most cases (Fig. 5).

The number of NOS-IR and VIP-IR nerves along the 

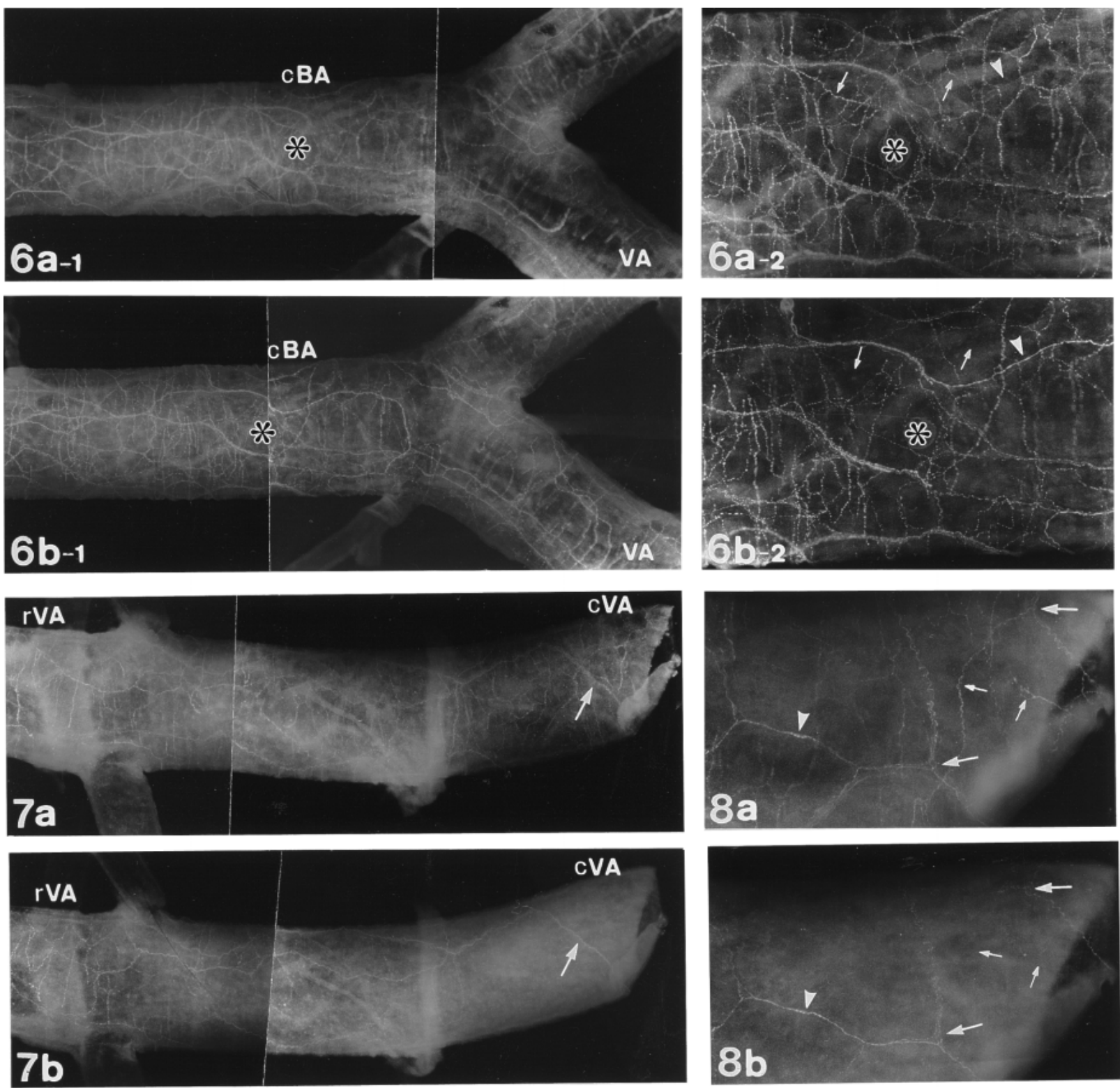

Figs. 6-8. Fluorescence photomicrographs of whole mounts with double staining for VIP (a) and NOS (b) in the basilar and vertebral arteries (BA, VA) on PND 30. Figure 6. Caudal part of the BA (cBA) and the rostral part of the VA (rVA). Arrows indicate VIP-IR nerves negative for NOS. Arrowheads indicate NOS-IR nerves negative for VIP. Asterisks indicate the same portion of the cBA. $\times 50(6 \mathrm{a}-1,6 \mathrm{~b}-$ $1), \times 100(6 a-2,6 b-2)$. Figure 7. Caudal and rostral parts of the VA (cVA, rVA). Arrows indicate a fiber bundle immunoreactive for both VIP and NOS. Note that NOS-IR and VIP-IR fiber bundle in the cVA sends its fiber branches to the rVA. $\times 50$. Figure 8 . Caudal part of the VA. Small arrows indicate VIP-IR nerves negative for NOS. Note the appearance of fiber bundles (large arrows) and axons (arrowheads) immunoreactive for both VIP and NOS. $\times 100$.

cBA and the VA increased distinctly after the third postnatal week. Thus, in most of the vascular preparations examined at PND 30, a moderate or rather much number of $\mathrm{NOS}^{+} /$ $\mathrm{VIP}^{+}, \mathrm{NOS}^{+} / \mathrm{VIP}^{-}$and $\mathrm{VIP}^{+} / \mathrm{NOS}^{-}$nerves was distributed in a network-like fashion along the cBA (Figs. 6, 7). The rVA was also furnished with a similar or rather less density of these three types of nerves. The nerve supply, however, became sharply poor in the cVA (Figs. 8,9 ): there was a dis- tinct decline in number of $\mathrm{NOS}^{+} / \mathrm{VIP}^{+}$or $\mathrm{NOS}^{+} / \mathrm{VIP}^{-}$nerves.

In the cBA of the adult rats aged at PND 80, NOS-IR and VIP-IR nerves tended to increase further in density (Fig. 10). Here, it was seen with high frequency that relatively well-organized plexuses were formed by a mixture of nerves stained doubly or singly for NOS and VIP. Similarly, the VA was almost entirely surrounded by a substantial amount of these nerve types. 

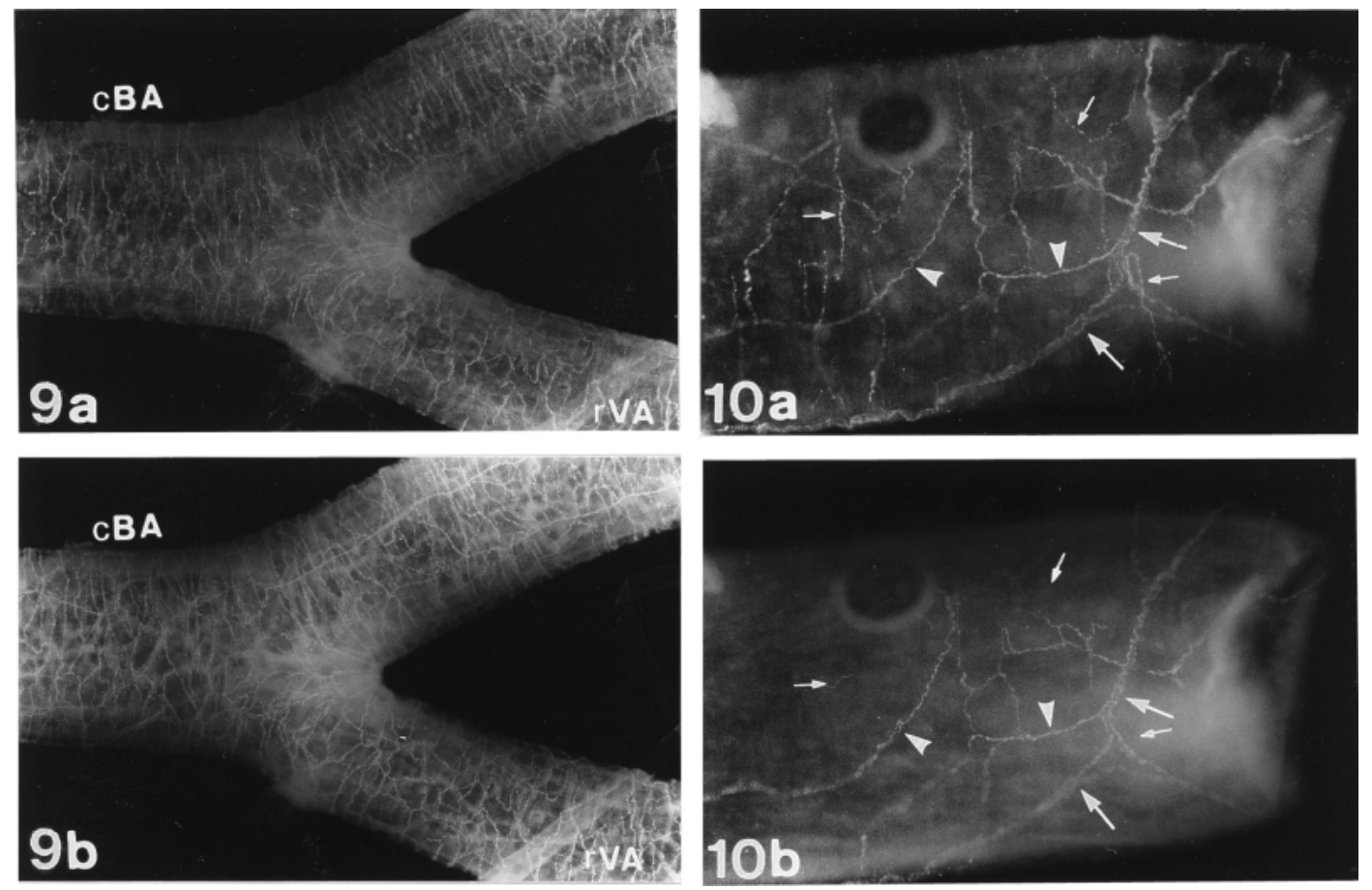

Figs. 9, 10. Fluorescence photomicrographs of whole mounts with double staining for VIP and NOS in the basilar and the vertebral arteries (BA, VA) on PND 80. Figure 9. Caudal part of the BA (cBA) and rostral part of the VA (rVA). $\times 50$ Figure 10. Caudal part of the VA. Small arrows indicate VIP-IR nerves negative for NOS. Note the presence of fiber bundles (large arrows) and axons (arrowheads) immunoreactive for both VIP and NOS. $\times 100$.

In the vascular preparations at PND 30, one or two fiber bundles containing NOS-IR axons, which were mostly immunoreactive for VIP, were occasionally seen limited to the cVA (Figs. 8, 9). The localization of such fiber bundles could not be demonstrated in the VA at any stages of development before PND 30, but were frequently encountered in the corresponding arterial region at PND 80 (Fig. 10).

\section{DISCUSSION}

Evidence that the principal site of origin for cerebrovascular NOS-IR and VIP-IR innervations is the SPG has been provided by immunohistochemical studies in adult rats [7, $11,12,14,16]$. The axons from here pass through the ethmoidal foramen, enter the cranial cavity along the IEA as fiber bundles, and then attain as far as the level of the mBA caudally. NOS-IR and VIP-IR neurons from another two parasympathetic ganglia, the internal carotid mini-ganglion and the otic ganglion, also project, to some extent, to the circle of Willis through the CCA, but do not reach caudally to the BA $[5,11,12]$. Our previous ontogenical studies, which are carried out using single staining, have revealed that NOS-IR and VIP-IR neuronal projections from the anterior circulation to the mBA resemble strikingly VIP-IR ones in the developmental pattern, being usually accomplished at the beginning of the third postnatal week $[2,3]$. This observation is confirmed and extended by the present double immunohistochemistry showing that at the PND 15, the rostral half of the BA is almost all covered by a moderate number of $\mathrm{NOS}^{+} / \mathrm{VIP}^{+}$nerves, whereas its caudal half has in most cases no supply of nerves with detectable level of NOS immunoreactivity. Minami et al. [11] have pointed out that not all NADPHd-positive nerves supplying the rat cerebral arterial tree are immunoreactive for VIP and vice versa. The same was true for cerebrovascular NOS-IR and VIP-IR innervations in the major cerebral arteries located more rostral than the mBA: a few $\mathrm{NOS}^{+} / \mathrm{VIP}^{-}$or $\mathrm{VIP}^{-} / \mathrm{NOS}^{+}$nerves are intermingled among $\mathrm{NOS}^{+} / \mathrm{VIP}^{+}$nerves.

Bilateral extirpation of the adult rat SPG, showing no discernable effect on the degree of NOS-IR and VIP-IR innervations in the cBA and the VA, suggests the possibility that NOS-IR and VIP-IR nerves supplying these arterial regions, if not all, have their source at another ganglia discrete from the SPG and come from the VA [11-13]. In the present study, the walls from the cBA to the VA are completely devoid of $\mathrm{NOS}^{+} / \mathrm{VIP}^{+}$or $\mathrm{NOS}^{+} / \mathrm{VIP}^{-}$nerves on PND 10 . Even in the third postnatal week (PND 15 and 20), NOS-IR nerves are mostly absent from the cVA or very sparse if they exist. Consequently, we have clearly demonstrated that VIP-IR nerves projecting to the BA from the VA, which are consistently found all through the postnatal stages investigated, almost all express no NOS immunoreactivity before the fourth postnatal week. In addition to the observations mentioned above, fiber bundles containing $\mathrm{NOS}^{+} / \mathrm{VIP}^{+}$ 
axons first appear on the cVA at PND30. The appearance of such fiber bundles is visualized more frequently on this vessel wall in the adult rats aged at PND 80, with an increase in density of NOS-IR and VIP-IR nerves supplying the cBA and the VA. Thus, the combination of these findings makes it possible to speculate that the projection of NOS-IR nerves to the cerebral arterial tree from the VA generally starts after the fourth postnatal week, being distinctly far late in the time of appearance when compared to VIP-IR neuronal projection from the same vascular route and NOS-IR and VIPIR neuronal projection from the anterior circulation to the BA.

In conclusion, the present study has provided immunohistochemical evidence that NOS-IR and VIP-IR neurons projecting from the VA to the cerebral arterial tree in the rat develop through their own characteristic sequences that are defined neurochemically. It remains to be settled whether the nitrergic and VIPergic neurons questioned are contained in the same or separate ganglia, being probably some parasympathetic ones that are located more caudal than the SPG.

\section{REFERENCES}

1. Ando, K. 1998. Nitrergic innervation of the cerebral arterial tree in the bent-winged bat (Mammalia: Microchiroptera). $J$. Comp. Neurol. 390: 366-376.

2. Ando, K., Ishikawa, A., Kawakami, H. and Sakuma. Y. 1991. Development of AChE-positive, NA-containing and VIP-and NPY-immunoreactive nerves in the major cerebral arteries of the rat. Anat. Embryol. 184: 25-32.

3. Ando, K., Mishima, Y. and Sakai, M. 2004. Development of nitric oxide synthase-immunoreactive nerves in the cerebral arteries of the rat. J. Vet. Med. Sci. 66: 933-940.

4. Barroso, C. P., Edvinsson, L., Zhang. W., Sa, M. Ce. Springall, D. P., Polak, J. M. and Gulbenkian, S. 1996. Nitroxidergic innervation of guinea pig cerebral arteries. J. Auton. Nerv. Syst. 58: $108-114$.

5. Bleys, R. J. A. W. and Cowen, T. 2001. Innervation of cerebral blood vessels: Morphology, plasticity, age-related, and Alzheimer's disease related neurodegeneration. Microsc. Res. Tech. 53: $106-118$.

6. Chen, F.-Y. and Lee, T. J.-F. 1993. Role of nitric oxide in neu- rogenic vasodilatation of porcine cerebral artery. J. Pharmacol. Exp. Ther. 264: 339-345.

7. Edvinsson, L., Elsas, T., Suzuki, N., Shimizu, T. and Lee, T. J. -Y. 2001. Origin and co-localization of nitric oxide synthase, CGRP, PACAP, and VIP in the cerebral circulation of the rat. Microsc. Res. Tech. 53: 221-228.

8. Ignacio, C. S., Curling, P. E., Childres, W. F. and Bryan, Jr. Y. 1997. Nitric oxide-synthesizing perivascular nerves in the rat middle cerebral artery. Am. J. Physiol. 273: R661-R668.

9. Kimura, T., Yu, T.-G., Edvinsson, L. and Lee, T. J.-F. 1997. Cholinergic, nitric oxidergic innervation in cerebral arteries of the cat. Brain Res. 773: 117-124.

10. Lee, T. J.-F. and Sarwinski, S. J. 1991. Nitric oxidergic neurogenetic vasodilation in the porcine basilar artery. Blood Vessels 28: 407-412.

11. Minami, Y., Kimura, H., Aimi, Y. and Vincent, S. R. 1994. Projection of nitric oxide-synthase containing fibers from the sphenopalatine ganglion to cerebral arteries in rat. Neuroscience 60: 745-759.

12. Nozaki, K., Moskowizt, M. A., Maynard, K. I., Koketsu, N., Dawson, T. M., Bredt, D. S. and Snyder, S. H. 1993. Possible origin and distribution of immunoreactive nitric oxide synthase-containing nerve fibers in cerebral arteries. J. Cereb. Blood Flow Metab. 9: 70-79.

13. Suzuki, N., Hardebo, J. A. and Owman, C. 1988. Origins and pathways of cerebrovascular vasoactive intestinal polypeptidepositive nerves in rat. J. Cereb. Blood Flow Metab. 8: 697712.

14. Suzuki, N., Fukuuchi, Y., Koto, A., Naganuma, Y., Isozumi, K., Konno, S., Gotoh, J. and Shimizu, T. 1994. Distribution and origins of cerebrovascular NADPH-diaphorase-containing nerve fibers in the rat. J. Auton. Nerv. Syst. 49: S51-S54.

15. Toda, N. and Okumura, T. 1992. Regulation of nitrooxidergic nerves of arterial tone. News Physiol. Sci. 7: 148-152.

16. Young, H. M., Anderson, C. A. and Furness, J. B. 2000. Nitric oxide in the peripheral autonomic nervous system. pp. 215265. In: Functional Neuroanatomy of the nitric oxide system (Steinbusch, H. W. N., De Vente, J. and Vincent, S. R. eds.), Elsevier, Amsterdam.

17. Yu, J.-G., Kimura, T., Chang, X.-F. and Lee, T. J.-F. 1998. Segregation of VIPergic and cholinergic-nitric oxidergic innervation in porcine middle cerebral arteries. Brain Res. 801: 7887. 